\title{
1 How the mitoprotein-induced stress response safeguards the cytosol: A unified view
}

2 Felix Boos ${ }^{1}$, Johnathan Labbadia ${ }^{2 *}$, Johannes M. Herrmann ${ }^{1 *}$

1, Cell Biology, University of Kaiserslautern, Kaiserslautern, Germany

42 2, Institute of Healthy Ageing, Department of Genetics, Evolution and Environment, University

5 College London, London, United Kingdom

6

7

8

9

*Correspondence: hannes.herrmann@biologie.uni-kl.de (J.M. Herrmann), j.labbadia@ucl.ac.uk (J. Labbadia)

\section{Keywords}

Proteostasis - Mitochondria - Protein Import - Heat Shock Factor 1 - Stress Response - Ageing

\section{Abstract}

Mitochondrial and cytosolic proteostasis are of central relevance for cellular stress resistance and organismal health. Recently, a number of individual cellular programs were described which counter the fatal consequences of mitochondrial dysfunction. These programs remove arrested import intermediates from mitochondrial protein translocases, stabilize protein homeostasis within mitochondria and, in particular, increase the levels and activity of chaperones and the proteasome system in the cytosol. Here, we describe the different responses to mitochondrial perturbation, and propose to unify the seemingly distinct mitochondrial-cytosolic quality control mechanisms into a single network, the mitoprotein-induced stress response. This holistic view places mitochondrial biogenesis at a central position of the cellular proteostasis network, emphasizing the importance of mitochondrial protein import processes for development, reproduction and ageing. 
The emerging role of mitochondria in the regulation of cellular and organismal protein homeostasis

Organization of the subcellular environment into distinct, membrane-bound organelles is a key feature of eukaryotic cells. While this allows cells to operate efficiently through the creation of functionally specialized environments, the spatial and temporal separation of protein synthesis, folding and degradation also presents a significant challenge to the cells' ability to maintain protein homeostasis (proteostasis).

In order to counteract proteostasis imbalances within compartments, cells have evolved dedicated, organelle-specific protein quality control programs, such as the heat shock response (HSR)(see glossary) of the cytosol and the unfolded protein responses of the endoplasmic reticulum (UPR $\left.{ }^{\mathrm{ER}}\right)$ and mitochondria $\left(\mathrm{UPR}^{\mathrm{mt}}\right)$ [1-3]. These responses have been extensively studied, and are crucial for the functionality of cells, tissues and organisms. However, the classical view that organellar stress responses act in isolation has been challenged by observations in yeast, worms, flies and mammalian tissue culture cells. Rather, proteotoxic insults at the organellar level can have farreaching consequences for protein quality control networks across the cell, or even for other tissues [4-6]. This has become particularly clear in the case of mitochondria, where the activity and composition of cytosolic proteostasis networks is tightly coordinated with fluctuations in mitochondrial activity and function, through several seemingly distinct protective responses. In this review, we present the different pathways that couple changes in mitochondrial function with cytosolic protein homeostasis, and discuss how these seemingly disparate mechanisms might be integrated into one coordinated mitoprotein-induced stress response that impacts development, ageing and disease. 
Mitochondria are responsible for the bulk of cellular ATP production, and are classically referred to as the 'powerhouses' of the cell. Interestingly, a growing number of studies have connected mitochondrial function with susceptibility to, and protection against, cytosolic protein aggregation [7-13]. Impaired cell function as a consequence of mitochondrial dysfunction was initially attributed to changes in the levels of ATP or reactive oxygen species (ROS); however, another factor might be of even more direct relevance: the integrity of the mitochondrial protein import process [14].

Only a few hydrophobic core subunits of the respiratory chain (and a ribosomal protein in yeast) are encoded in the mitochondrial genome and produced within mitochondria. The other approximately 1000 mitochondrial proteins are synthesized on cytosolic ribosomes, subsequently targeted to the mitochondrial surface and then imported by dedicated translocases (Figure 1) [15]. Most mitochondrial proteins are synthesized as precursors with an $\mathrm{N}$-terminal mitochondrial targeting sequence (MTS, also called presequence) which are cleaved upon arrival in the mitochondrial matrix. Mitochondrial functionality relies on an efficient protein import process and translate into import defects (Figure 1).

In addition, protein import is sensitive to precursor state and load: The excessive synthesis of precursors, or stalling of prematurely folded import intermediates within translocases, can cause import defects [16-19]. In particular, proteins which are $\mathrm{N}$-terminally anchored to the inner membrane are difficult to import due to the presence of stop-transfer signals after their 
Owing to their post-translational mode of import, mitochondrial precursors are transiently exposed to the cytosol, where they are stabilized by chaperones [22-26] and under the surveillance of the proteasomal degradation system [27-29]. The passage of precursors through the cytosol makes the import process vulnerable to proteotoxic insults outside of mitochondria. In fact, cytoplasmic aggregation of pathological protein species such as mutant huntingtin/polyQ proteins [30-32], $\alpha$ synuclein [33, 34] or amyloid $\beta[9,35-37]$ all interfere with mitochondrial protein import. Moreover, it was suggested that aggregated proteins in the cytosol are imported into mitochondria for sequestration or subsequent degradation [38, 39], although the underlying mechanism and relevance of this pathway is under debate. Together, these observations place mitochondrial protein import at the center of cellular proteostasis networks, well beyond the mitochondrial compartment (Figure 2).

\section{Consequences of impaired mitochondrial protein import}

If mitochondrial protein import is defective, cells face two major challenges. On the one hand, the lack of protein supply leads to proteome imbalances inside mitochondria, comparable to consequences of defects in the expression of the mitochondrial genome [40]. On the other hand, import defects result in the accumulation of precursor proteins in the cytosol and challenge proteostasis outside mitochondria.

It has been estimated that under normal basal conditions, around 5\% of nascent ER proteins might constitutively fail to reach the ER at steady state conditions [41]. A similar magnitude also seems likely for mitochondrial preproteins, especially because some mitochondrial precursor proteins can traverse the ER surface on their route to mitochondria [42]. Small amounts of orphaned proteins can be efficiently cleared from the cytosol by proteasomal degradation [27] or from membranes by more specific mechanisms that degrade or re-route mislocalized proteins [43, 44]. However, when mitochondrial protein import efficiency is globally reduced, the fraction of accumulating precursors 
can increase substantially, thereby placing a burden on protein folding and degradation pathways. As pre-proteins are escorted to the mitochondrial translocases by chaperones of the HSP70, HSP90 and HSP40 families [22, 23], a higher load of precursors could sequester these chaperones, leading to reduced protein folding capacity in the cytosol. In addition, most mitochondrial proteins are unlikely to fold properly outside mitochondria and can associate with, and perhaps even induce, cytosolic aggregates $[45,46]$. Thus, defects in the import of mitochondrial precursors induce a situation that is reminiscent of the widespread decline of proteostasis that is associated with protein conformational diseases or ageing $[47,48]$.

\section{Cellular reactions to compromised mitochondrial protein import}

Cells use a repertoire of means to prevent an overload of mitochondrial protein import and to counteract the consequences of import failure for both mitochondria and the cytosol. Initially described as individual phenomena, numerous studies have revealed that cells safeguard mitochondrial protein import and restore mitochondrial/cytosolic homeostasis by: (1) unclogging jammed translocases and removing accumulating precursor proteins from the mitochondrial surface $[19,20]$; (2) adjusting the synthesis of mitochondrial proteins to match import capacity, and increasing the expression of mitochondrial biogenesis and quality control components to preserve mitochondrial integrity [18, 49]; and (3) engaging cytosolic protein folding and degradation machineries to relieve the burden of accumulating precursor proteins outside of mitochondria $[18,50]$ (Figure 2). These mechanisms have been described in different organisms using several experimental models, such as mutants of the mitochondrial import machinery [29, 50-53], overexpression of proteins whose translocation is challenging [17-20], and disruption of mitochondrial membrane potential [54-57]. 
125

126

127

To prevent clogging of the import channel by non-productive import intermediates, the translocase of the outer mitochondrial membrane (TOM) is continuously monitored by the mitochondrial protein translocation-associated degradation (mitoTAD) pathway. In yeast, the key component of this pathway is Ubx2, which also functions in ER-associated degradation (ERAD). Ubx2 is part of the TOM complex; upon the appearance of arrested precursors in the translocase, Ubx 2 recruits the AAA ATPase Cdc48/VCP/p97 to extract trapped precursors and direct them to the proteasome for degradation [19].

In addition, the mitochondria-associated AAA ATPase Msp1 monitors the complete mitochondrial surface for aberrant protein species. Msp1 recognizes tail-anchored membrane proteins that are mistargeted to mitochondria, extracts them from the mitochondrial outer membrane and re-routes them to the ER [43]. In addition, upon blocked mitochondrial import, the adaptor protein Cis 1 is expressed which recruits Msp1 to the TOM complex. There, Msp1 and Cis1 mediate the removal of the precursor proteins, a process known as the mitochondrial compromised protein import response (mitoCPR) [20].

Besides premature folding or weak translocation, precursor proteins can also arrest inside translocases due to stalling of the ribosome during translation. In the cytosol, arrested ribosomenascent chain complexes are cleared by dedicated ribosome quality control (RQC) pathways, involving the addition of C-terminal amino acids to the stalled polypeptide (CAT tailing) to facilitate its degradation by the ubiquitin-proteasome system. However, when the ribosome-nascent chain complex associates with the mitochondrial import machinery, CAT-tailed polypeptides are no longer accessible to the cytosolic quality control machinery and tend to aggregate inside mitochondria [58]. The conserved quality control factor Vms1 recognizes ribosome-stalled proteins at the mitochondrial surface and prevents CAT tailing [59-61]. In addition to its role in this mitochondrial RQC pathway, Vms1 also recruits Cdc48 to mitochondria upon stress to assist with protein degradation [57]. 
151 Imbalances in the mitochondrial proteome are counteracted by a transcriptional program known as

152 the mitochondrial unfolded protein response $\left(\mathrm{UPR}^{\mathrm{mt}}\right)$. In a nutshell, protein import overload is 153 prevented by three major measures: (1) Increased expression of mitochondrial chaperones, 154 assembly factors and proteases [62]; (2) increased expression of mitochondrial translocases (in 155 metazoa, not in yeast) [63]; and (3) reduced expression of many mitochondrial proteins, particularly 156 the highly abundant enzymes of the respiratory chain and TCA cycle, the coordinated 157 downregulation of which, presumably relieves the workload of the import machinery [49]. Similar 158 to the role of the $\mathrm{UPR}^{\mathrm{ER}}$ in homeostatic regulation of ER size, the responsiveness of the expression 159 of mitochondrial enzymes to import overloading constitutes an elegant feedback mechanism to monitor and adjust the influx of proteins into mitochondria (Box 1).

The analysis of the $\mathrm{UPR}^{\mathrm{mt}}$ was pioneered by studies in C. elegans and has been extensively reviewed elsewhere [1]. The master regulator of the UPR ${ }^{\mathrm{mt}}$ in C. elegans is the transcription factor ATFS-1, which is a dually localized protein, present in mitochondria and the nucleus. A weak MTS efficiently targets ATFS-1 to mitochondria in well energized cells. However, when mitochondrial functions are compromised, ATFS-1 is no longer imported into mitochondria but instead accumulates in the nucleus, where it induces the $\operatorname{UPR}^{\mathrm{mt}}[55,63]$. In human cells, the transcription factors ATF4 and ATF5 were proposed to fulfill a similar role $[64,65]$. Yeast does not contain ATFS-1 homologs, but the HAP complex, which regulates the expression of most respiratory components, appears to play a comparable role. HAP-regulated genes are repressed upon protein import overload by inactivation of this transcription factor complex [18]. However, the underlying molecular mechanisms still remain to be discovered. 
The accumulation of mitochondrial precursors in the cytosol is buffered by an upregulation of many cytosolic chaperones, including members of the HSP70, HSP90, HSP40, TRiC/CCT, and small heat shock protein families $[18,53,66]$. In addition, the abundance and activity of the proteasome is increased in a reaction known as the unfolded protein response activated by mistargeting of proteins (UPRam) $[18,50]$ or mitochondrial precursor over-accumulation stress (mPOS) [54]. The elevated proteasomal capacity helps to remove precursors from the cytosol and assists in the clearance of the outer membrane in conjunction with the mitoTAD and mitoCPR pathways. Although the protective responses described above were discovered as independent phenomena, recent evidence suggests that in fact, these pathways are amalgamated into a collective protective program, the mitoproteininduced stress response [18](Figure 3, Key Figure).

The transcription factor HSF1 is crucial for maintaining proteostasis in the cytosol and has emerged as a key component of the mitoprotein-induced stress response. HSF1 dictates protein folding and degradation capacity in the cytosol through the coordinated expression of molecular chaperones, co-chaperones and degradation factors. It has long been known that in yeast, the transition from fermentative to respiratory metabolism, which strongly induces the production of mitochondrial proteins, is accompanied by an HSF1-mediated upregulation of chaperones and other stressresponsive factors $[67,68]$. Consistent with this, it was recently discovered that mitochondrial import stress, impaired respiration or perturbation of mitochondrial HSP70, leads to a rapid elevation in the levels of HSF1 target genes [18, 66, 69], thereby augmenting the function of the core cytosolic proteostasis network.

Under non-stress conditions, HSF1 activity is repressed by direct interactions with molecular chaperones. However, upon proteostasis imbalances in the cytosol, molecular chaperones are titrated away from HSF1 through preferential binding to misfolded protein species. This permits the activation of functional HSF1 heterotrimers, and results in the increased expression of genes that restore cytosolic proteostasis $[70,71]$. It is highly likely that the accumulation of unstable 
mitochondrial precursors in the cytosol triggers the activation of HSF1 through a similar mechanism. However, it remains unclear whether HSF1 activation results from a general overload of the cytosol by mitochondrial precursors, or whether specific (groups of) precursors trigger HSF1 activation. Since the signatures of mitoprotein-induced stress response and heat shock response are similar but not identical, it is possible that additional mechanisms tailor chaperone expression to the specific sources of misfolded proteins. For example, lipid signaling has been reported to activate HSF1 upon mitochondrial perturbation in nematodes and thus might represent an addition layer of regulation [66].

In yeast, HSF1 also promotes the expression of Rpn4, the master regulator of proteasomal subunits and components of the ubiquitin proteasome system (UPS). [72]. The transcriptional induction of Rpn4 by Hsf1 is responsible for the upregulation of the ubiquitin-proteasome system in response to mitoprotein-induced stress [18]. Rpn4 itself is also a substrate of proteasomal degradation with very efficient turnover. Therefore, occupancy of the proteasome by mitochondrial precursors might also directly lead to the stabilization and, hence, increased abundance of Rpn4, augmenting its transcriptional upregulation.

In addition to proteasomal subunits, Rpn4 also increases the expression of Ubx2 and Cdc48, the central mediators of mitoTAD, and the transcription factor Pdr3, which in turn drives the expression of the mitoCPR factor Cis1 [20]. Therefore, HSF1 acts as the primary initiator of an Hsf1-Rpn4Pdr3 transcriptional cascade that directly connects the regulation of mitochondrial and cytosolic responses to proteotoxic stress. The Hsf1-Rpn4-Pdr3 transcriptional axis is an intriguing example of how cells can coordinate the activity of multiple stress-related regulators. This mechanism has clear similarities with how increased Rpn4 levels in response to impaired translocation of ER preproteins, complements the $\mathrm{UPR}^{\mathrm{ER}}$ to maintain cell viability [73]. This suggests that cells have evolved a general 'core' response to protein misfolding in the cytosol that is converged upon by mistargeted proteins or proteins that are misfolded due to heat exposure or other stresses. 
228

229

The transcriptional response to mitoprotein-induced stress is accompanied by the attenuation of protein synthesis $[18,50,51,54,74]$. This decreases the load on both the cytosolic protein quality control and mitochondrial import machineries, and saves energy. In addition to the specific shutdown of the synthesis of mitochondrial OXPHOS components, translational attenuation further reduces the production of mitochondrial precursors. Moreover, gene expression from the mitochondrial genome is also repressed $[18,75]$. This may help to balance protein synthesis in the matrix with the reduced influx of imported proteins.

Reduced cytosolic translation has been proposed to occur through transcriptional downregulation and reversible cysteine oxidation of $80 \mathrm{~S}$ ribosomal subunits. While still speculative, this suggests a model where mitochondrial stress can directly alter cytosolic translation through 'redox switches' in ribosomal subunits [51]. In addition, protein synthesis can be reduced through eIF2 $\alpha$ phosphorylation as part of the integrated stress response and through inhibition of the target of rapamycin (mTOR) complex. Both eIF2 $\alpha$ phosphorylation and reduced mTOR activity have been observed in response to mitochondrial stress [76, 77]; however, the precise contribution of these pathways to mitoprotein-induced slowdown of translation remains to be determined.

\section{Conservation of the mitoprotein-induced stress response}

Although well-described in yeast, the regulatory basis and composition of the mitoprotein-induced stress response in metazoans is less well understood. However, available evidence suggests that analogous mechanisms to those observed in fungi are present in animals. For example, the targeting of misfolding-prone substrates to mitochondria, genetic and chemical inhibition of respiration and perturbation of mitochondrial HSP70 have all been reported to increase the expression of HSF1 target genes in C. elegans and Drosophila [56, 66, 69, 78]. 
In addition to the immediate activation of acute transcriptional responses, mitochondrial status has also emerged as a critical determinant of HSF1 activity and susceptibility to protein aggregation later in adulthood. In $C$. elegans, the transition to reproductive maturity is accompanied by the programmed repression of the heat shock response [79]. This is mediated by changes in chromatin architecture at HSF-1 target promoters and leaves cells vulnerable to protein folding stress later in life. Mild perturbation of either respiration or mitochondrial import efficiently maintains the activity of the heat shock response in aged animals and protects against age-related protein aggregation, suggesting that exposure to mitochondrial stress can override age-related changes in chromatin organization and the heat shock response [56]. Although the precise mechanism by which mitochondrial impairment maintains the heat shock response is unknown, mitochondrial stress and full activation of the UPR ${ }^{\mathrm{mt}}$ are also associated with changes in chromatin organization $[78,80,81]$ (Box 2). Together, these observations demonstrate the existence of a complex link between mitochondrial function, chromatin organization, HSF1 activity, cytsolic proteostasis and ageing.

While HSF1 activity is clearly linked to mitochondrial function in worms and flies, the regulation of the proteasome under mitoprotein-induced stress is far less clear in animals, particularly as orthologues of Rpn4 do not exist in metazoans. Potentially, the transcription factors NRF1 and NRF2 could fulfill a similar role as Rpn4. Like Rpn4, NRF1 and NRF2 control the abundance of proteasomal subunits in response to compromised proteasome activity. NRF2 has been shown to localize to the surface of mitochondria and is activated by mitochondrial ROS upon proteasome dysfunction [82, 83]. Furthermore, the C. elegans orthologue of NRF1, SKN-1A, promotes a UPRam-like cytoplasmic unfolded protein response to counteract various proteotoxic stresses [84]. Thus, the general regulatory principles appear to be conserved among eukaryotes. These make sure that upon mitoprotein-induced stress, proteostatic balance is maintained in both the cytosol and the mitochondria. This response employs regulators of general stress programs, in particular those of the heat shock response, as well as mechanisms that act at the level of specific steps of mitochondrial protein synthesis and import. Dependent on the severity and duration of 
mitochondrial defects, the mitoprotein-induced stress response is also coupled with more global cellular homeostatic programs, which employ processes such as chromatin re-organization [56, 81] autophagy/mitophagy [85-87] and apoptosis [21] to promote transcriptional responses, signal to unaffected tissues, remove defective mitochondria or eliminate unviable cells (Figure 4).

Even though the general principles of these programs appear to be similar among eukaryotes, a considerable amount of heterogeneity exists with respect to the specific factors and regulatory elements that drive these programs in different organisms. One obvious example is that the expression of mitochondrial proteins is muted in nematodes by the stress response factor ATFS-1, whereas in yeast this is controlled by the general respiration control complex HAP. As such, understanding why these differences have emerged may provide important insight regarding the coordination of mitochondrial and cytosolic proteostasis across developmental states and/or cell types.

\section{Concluding Remarks}

Over the last five years, it has become increasingly evident that cellular stress resistance and organismal health are highly dependent on connections between mitochondrial and cytosolic proteostasis. While not all connections and causalities are understood (see Outstanding Questions Box), two major paradigms have emerged: First, the proteostasis and quality control programs from different subcellular compartments are distinct, but do not act in isolation from each other. Second, many seemingly disparate mechanisms are wired into a coordinated network that simultaneously restores mitochondrial function and safeguards cytosolic proteostasis. Therefore, we propose to amalgamate the existing independent mitochondrial-cytosolic quality control mechanisms into a single network, the mitoprotein-induced stress response. Taking a more holistic view of the mitochondrial-cytosolic protein quality control network will allow us to unravel the full complexity 
301 of how mitochondrial function is coordinated with alterations in proteostasis and how this impacts

302 development, reproduction and ageing.

303

304 Acknowledgements

305 We thank Katharina Knöringer, Lena Krämer, Carina Groh and Jana Friedl for helpful discussions 306 and valuable comments on the manuscript. Authors were supported by funding from the Deutsche 307 Forschungsgemeinschaft (DIP MitoBalance, IRTG1830 and the SPP1710 to J.M.H.), the 308 Forschungsinitiative Rheinland Pfalz BioComp (to J.M.H.), the Joachim Herz Stiftung (to F.B.), a 309 BBSRC David Phillips Fellowship (to J.L.), an AMS Springboard Award (to J.L.) and a Wellcome 310 Trust Institutional Strategic Support grant (to J.L.). 
313 Protein misfolding in the lumen and the membrane of the ER is recognized by receptors located in

314 the ER membrane. Upon stress, these elicit signaling pathways which induce or repress genes to

315 buffer and counter problems within the ER. In contrast, the mitoprotein-induced stress response

316 reacts to the presence of mitochondrial proteins that fail to be efficiently imported. Signaling can

317 be triggered by specific stress-sensing factors, such as ATFS-1 of C. elegans, or by a more global

318 accumulation of mitochondrial precursors, as proposed by the UPRam hypothesis for yeast

\section{9 (Figure I).}

320 The distinction between transmembrane signaling from the ER and a "frustrated client" reporting

321 model from mitochondria is not "black and white": Non-imported ER proteins are sensed in the

322 cytosol $[73,88]$ and proteotoxic stress in the matrix of mitochondria can induce transcriptional

323 changes in the nucleus [11, 89]. However, for most mitochondrial stress responses, signaling

324 seems to occur mainly at the level of preprotein import from the cytosol and not via direct

325 transduction across mitochondrial membranes. 


\section{through chromatin reorganization}

330

The rapid and effective activation of stress responsive transcriptional programs such as the HSR and $\mathrm{UPR}^{\mathrm{mt}}$ is crucial for cells to successfully counteract proteostasis imbalances. In addition to the activity of dedicated transcription factors, it has recently been demonstrated that in C. elegans, changes in histone methylation and chromatin remodeling are crucial for effective induction of the $\mathrm{UPR}^{\mathrm{mt}}$, maximal induction of HSF1 target genes and full lifespan extension when respiration is compromised [56, 69, 80, 81].

In response to mitochondrial stress, chromatin architecture is reorganized through the chromatin remodeler LIN-65 in a process that is dependent on MET-2-mediated di-methylation of lysine 9 of histone $\mathrm{H} 3$ (H3K9me2). This results in a global chromatin conformation that generally represses transcription while favoring the induction of $\mathrm{UPR}^{\mathrm{mt}}$ responsive genes [81]. Similarly, the HSF1mediated induction of small heat shock protein genes upon electron transport chain dysfunction is also dependent on chromatin remodeling through the SWI/SNF-related factor, ISW-1 [69].

In addition to promoting immediate responses through HSF1 and the UPR ${ }^{\mathrm{mt}}$, mitochondrial stressmediated changes in chromatin status can also promote long-term cell function. Upon electron transport chain perturbation, increased JMJD-1.2 and JMJD-3.1 activity results in reduced levels of di- and tri-methylation at lysine 27 of histone $\mathrm{H} 3$ (H3K27me2/3). This results in increased chromatin accessibility, prolonged activation of the UPR ${ }^{\mathrm{mt}}$ and increased lifespan [80]. JMJD-3.1 activity has also been linked with the programmed repression of the HSR during early C. elegans adulthood. As worms reach reproductive maturity, signals from germ line stem cells result in decreased jmjd-3.1 expression, increased levels of $\mathrm{H} 3 \mathrm{~K} 27 \mathrm{me} 3$, and reduced chromatin accessibility at HSF1 target promoters. This leads to a dampening of the HSR and increased vulnerability to protein aggregation later in life [79]. While jmjd-3.1 over-expression does not influence HSF1 activity early in life, it is sufficient to promote the UPR ${ }^{\mathrm{mt}}$, maintain HSF1 activity in aged cells and extend lifespan [79, 80]. Intriguingly, repression of the HSR and age-related cytosolic protein 
354 aggregation can be suppressed by exposure to mitochondrial stress early in life [56]. While it is not 355 clear to what extent these effects are mediated by altered histone modification and chromatin re356 organization, these observations suggest that mitochondrial function is intimately coupled with the 357 long and short-term activity of both HSF1 and the UPR ${ }^{\mathrm{mt}}$ through changes in chromatin state. 


\section{Figure legends}

360

361

362

363

364

365

366

367

Figure 1. Mitochondrial protein import is challenged upon many conditions. Mitochondrial

biogenesis requires the import of about 1,000 different proteins from the cytosol. About two thirds of these proteins are initially made as precursors with an N-terminal mitochondrial targeting sequence (MTS). These sequences are recognized by receptors on the mitochondrial surface (Tom70 and cytosol-exposed regions of the TOM complex) and direct precursor proteins through the protein-conducting channels of the TOM and TIM23 complexes. The membrane potential across the inner membrane $(\Delta \psi)$ and ATP hydrolysis by HSP70 drive protein translocation.

Proteins of the intermembrane space (IMS) and the outer membrane often lack N-terminal targeting sequences and use distinct import routes. Many IMS proteins contain cysteine residues and their import is associated with oxidative protein folding in the IMS, catalyzed by the oxidoreductase Mia40. There are different groups of outer membrane proteins, including poreforming $\beta$-barrel proteins and tail-anchored proteins. In most cases, the import of Mia40 substrates and outer membrane proteins requires neither ATP nor a membrane potential across the inner membrane. The figure illustrates these key steps of mitochondrial protein biogenesis. The import process can be challenged by problems in the cytosol or by mitochondrial defects, some of which are indicated here in light boxes.

Figure 2. Import defects threaten cytosolic proteostasis. Under physiological conditions, precursor proteins are hardly detectable in the cytosol as they are rapidly imported or degraded. However, adverse conditions can lead to a slow-down of the import process and the accumulation of non-productive translocation intermediates. These can be removed by different mechanisms. Precursor proteins that are stalled in the TOM complex are degraded by the proteasome in a process referred to as mitoTAD. Ubx2 serves as a bridging factor in this process, which connects the TOM complex to $\mathrm{Cdc} 48 / \mathrm{VCP} / \mathrm{p} 97$, in order to extract the precursors from the TOM channel 
and feed them to the proteasome. Missorted outer membrane proteins are recognized and extracted by Msp1, an AAA protein on the mitochondrial surface. Upon accumulation of translocation intermediates that are stalled in the TOM complex, Msp1 is recruited to Tom70 by the bridging factor Cis1. This process is called mitoCPR, and cooperates with mitoTAD-mediated TOM clearance. Ribosomes that are stalled on non-functional mRNAs, and thereby tethered to TOM complexes, are removed by a dedicated machinery, which employs the Cdc48 interactor Vms1, in a process called mitoRQC. If these measures on the mitochondrial surface fail, precursors accumulate in the cytosol, where they sequester chaperones and serve as substrates of the proteasome. If the level of cytosolic precursors exceeds the capacity of the chaperone and proteasome system, cytosolic proteostasis is challenged, leaving cells vulnerable to widespread protein aggregation.

Figure 3, Key Figure. Regulation of the mitoprotein-induced stress response. Mitoproteininduced stress is countered by a concerted action of several transcription factors. In C. elegans, ATFS-1 serves as a major factor in the UPR ${ }^{\mathrm{mt}}$, which mutes the synthesis of mitochondrial proteins to relieve the burden on the mitochondrial import machinery. In yeast the HAP complex plays a comparable role, although the mechanistic details of this are still unclear. The accumulation of precursors in the cytosol leads to an induction of the heat shock response, triggered by HSF1. This attenuates protein synthesis and induces the expression of chaperones. In yeast, HSF1 also induces Rpn4, which serves as master transcription factor for the proteasomeubiquitin system. NRF2 may play a comparable role in animals. Rpn4 also induces Pdr3, the transcription factor that induces components of the multidrug resistance response, and Cis1, which connects Msp1 to the TOM complex for mitoCPR-mediated TOM clearance. Thus, at least in yeast, the components that trigger the mitoprotein-induced stress response form a reaction cascade, which sequentially activates different programs to maintain proteostasis in both mitochondria and the cytosol. 
412 duration. Muting the expression of mitochondrial proteins by ATFS-1 is an elegant mechanism to 413 adapt the amounts of produced precursors to the capacity of the mitochondrial import machinery.

414 This ensures that HSF1 activation is only triggered once the level of precursors exceeds the import capacity. The heat shock response is triggered by the release of HSF1 from chaperones and tailored by chromatin re-organization at HSF1 target promoters. The modification of chromatin state may be particularly relevant for persistently occurring challenges as this may allow cells to respond more effectively to subsequent mitochondrial insults. If serious mitochondrial problems remain over longer periods of time, mitochondria are removed by autophagy/mitophagy and affected cells are eliminated by apoptosis. Both pathways can be triggered by incomplete translocation and, consequently, accumulation of effector proteins on the mitochondrial surface - PINK1 in the case of mitophagy [85], Nde1 in the case of apoptosis [21]. How these drastic reactions are connected to the mitoprotein-induced stress response still awaits to be unraveled.

Figure I for Box 1. Stress signaling from the ER and mitochondria

\section{Glossary}

ERAD: Endoplasmic reticulum-associated protein degradation. Mediates the removal of proteins from the ER lumen or membrane by proteasomal degradation.

HSR: Heat shock response. Signaling pathway that is induced by the accumulation of unfolded or misfolded proteins in the cytosol and/or nucleus. The HSR is triggered by exposure to high temperature but can be induced by any conditions that promote protein misfolding.

mitoRQC: Mitochondrial ribosome quality control. Mutated mRNAs can irreversibly arrest translating ribosomes. If these stalled translation intermediates are targeted to 
mitochondria, a dedicated machinery recognizes and dissociates them to release the ribosome and degrade the non-productive nascent polypeptides.

437

mitoCPR: Mitochondrial compromised protein import response. Extraction system to remove arrested import intermediates from the TOM complex. Cis1 (together with Tom70) recruits the AAA extractor Msp1 to the TOM complex for back-translocation of precursors into the cytosol.

mitoTAD: Mitochondrial protein translocation-associated degradation. Degradation system to remove stalled translation intermediates from the TOM complex. For protein degradation of precursor proteins, the bridging factor Ubx2 recruits $\mathrm{Cdc} 48$ and the proteasome to the outer membrane receptor Tom70.

mPOS: Mitochondrial precursor over-accumulation stress. Describes the toxic accumulation of mitochondrial inner membrane proteins in the cytosol of yeast cells.

MTS: Mitochondrial targeting sequence or presequence at the N-terminus of mitochondrial precursor proteins. In most cases, presequences are removed after the import reaction by the mitochondrial processing peptidase giving rise to a mature mitochondrial protein.

UPRam: Unfolded protein response activated by mistargeting of proteins. Signaling pathway that is induced by mitochondrial precursor proteins which accumulate in the cytosol.

UPR $^{\text {ER: }} \quad$ Unfolded protein response. Signaling pathway that is induced by the accumulation of unfolded or misfolded proteins in the lumen or the membrane of the endoplasmic reticulum (ER).

UPR ${ }^{\mathrm{mt}} \quad$ Mitochondrial unfolded protein response. Signaling pathway that is induced by the accumulation of unfolded or misfolded proteins in the mitochondrial matrix. 
458 ROS: Reactive oxygen species. Highly reactive molecules including superoxide, hydrogen

459

460

461 TOM: Translocase of the outer membrane of mitochondria. The central pore-forming

462

463

464

465 peroxide and hydroxyl radicals that are formed by electron transfer to oxygen. Are produced as byproducts by the mitochondrial respiratory chain. subunit Tom40 serves as general entry gate for mitochondrial precursor proteins. Receptors such as Tom70 and Tom20/22 recognize cytosolic precursors and direct them to Tom 40 . 


\section{References}

467 1. Naresh, N.U. and Haynes, C.M. (2019) Signaling and Regulation of the Mitochondrial Unfolded Protein Response. Cold Spring Harb Perspect Biol 11 (6).

2. Preissler, S. and Ron, D. (2019) Early Events in the Endoplasmic Reticulum Unfolded Protein Response. Cold Spring Harb Perspect Biol 11 (4).

3. Morimoto, R.I. (2011) The heat shock response: systems biology of proteotoxic stress in aging and disease. Cold Spring Harb Symp Quant Biol 76, 91-9.

4. Quiros, P.M. et al. (2016) Mitonuclear communication in homeostasis and stress. Nat Rev Mol Cell Biol 17 (4), 213-26.

5. Moehle, E.A. et al. (2019) Mitochondrial proteostasis in the context of cellular and organismal health and aging. J Biol Chem 294 (14), 5396-5407.

6. Durieux, J. et al. (2011) The cell-non-autonomous nature of electron transport chain-mediated longevity. Cell 144 (1), 79-91.

7. Wang, P. et al. (2019) TDP-43 induces mitochondrial damage and activates the mitochondrial unfolded protein response. PLoS Genet 15 (5), e1007947.

8. Tsvetkov, P. et al. (2019) Mitochondrial metabolism promotes adaptation to proteotoxic stress. Nat Chem Biol 15 (7), 681-689.

9. Sorrentino, V. et al. (2017) Enhancing mitochondrial proteostasis reduces amyloid-beta proteotoxicity. Nature 552 (7684), 187-193.

10. Zhou, C. et al. (2014) Organelle-based aggregation and retention of damaged proteins in asymmetrically dividing cells. Cell 159 (3), 530-42.

11. Suhm, T. et al. (2018) Mitochondrial Translation Efficiency Controls Cytoplasmic Protein Homeostasis. Cell Metab 27 (6), 1309-1322 e6.

12. $\mathrm{Wu}$, Z. et al. (2018) Mitochondrial unfolded protein response transcription factor ATFS-1 promotes longevity in a long-lived mitochondrial mutant through activation of stress response pathways. BMC Biol $16(1), 147$.

13. Schroeder, E.A. et al. (2013) Epigenetic silencing mediates mitochondria stress-induced longevity. Cell Metab 17 (6), 954-64.

14. Harbauer, A.B. et al. (2014) The protein import machinery of mitochondria-a regulatory hub in metabolism, stress, and disease. Cell Metab 19 (3), 357-72.

15. Pfanner, N. et al. (2019) Mitochondrial proteins: from biogenesis to functional networks. Nat Rev Mol Cell Biol 20 (5), 267-284.

16. Kintaka, R. et al. (2016) Cellular growth defects triggered by an overload of protein localization processes. Sci Rep 6, 31774.

17. Liu, Y. et al. (2019) Mitochondrial carrier protein overloading and misfolding induce aggresomes and proteostatic adaptations in the cytosol. Mol Biol Cell 30 (11), 1272-1284.

18. Boos, F. et al. (2019) Mitochondrial protein-induced stress triggers a global adaptive transcriptional programme. Nat Cell Biol 21 (4), 442-451.

19. Martensson, C.U. et al. (2019) Mitochondrial protein translocation-associated degradation. Nature 569 (7758), 679-683.

20. Weidberg, H. and Amon, A. (2018) MitoCPR-A surveillance pathway that protects mitochondria in response to protein import stress. Science 360 (6385).

21. Saladi, S. et al. (2019) The NADH Dehydrogenase Nde1 Executes Cell Death after Integrating Signals from Metabolism and Proteostasis on the Mitochondrial Surface. Mol Cell.

22. Opalinski, L. et al. (2018) Recruitment of Cytosolic J-Proteins by TOM Receptors Promotes Mitochondrial Protein Biogenesis. Cell Rep 25 (8), 2036-2043 e5.

23. Young, J.C. et al. (2003) Molecular chaperones Hsp90 and Hsp70 deliver preproteins to the mitochondrial import receptor Tom70. Cell 112 (1), 41-50.

24. Gamerdinger, M. et al. (2015) The principle of antagonism ensures protein targeting specificity at the endoplasmic reticulum. Science 348 (6231), 201-7.

25. Doring, K. et al. (2017) Profiling Ssb-Nascent Chain Interactions Reveals Principles of Hsp70-Assisted Folding. Cell 170 (2), 298-311 e20. 
26. Stein, K.C. et al. (2019) Nascent Polypeptide Domain Topology and Elongation Rate Direct the Cotranslational Hierarchy of Hsp70 and TRiC/CCT. Mol Cell.

27. Kowalski, L. et al. (2018) Determinants of the cytosolic turnover of mitochondrial intermembrane space proteins. BMC Biol 16 (1), 66.

28. Mohanraj, K. et al. (2019) Inhibition of proteasome rescues a pathogenic variant of respiratory chain assembly factor COA7. EMBO Mol Med 11 (5).

29. Paasch, F. et al. (2018) Failed mitochondrial import and impaired proteostasis trigger SUMOylation of mitochondrial proteins. J Biol Chem 293 (2), 599-609.

30. Yablonska, S. et al. (2019) Mutant huntingtin disrupts mitochondrial proteostasis by interacting with TIM23. Proc Natl Acad Sci U S A 116 (33), 16593-16602.

31. Yano, H. et al. (2014) Inhibition of mitochondrial protein import by mutant huntingtin. Nat Neurosci 17 (6), 822-31.

32. Bauerlein, F.J.B. et al. (2017) In Situ Architecture and Cellular Interactions of PolyQ Inclusions. Cell 171 (1), 179-187 e10.

33. Szego, E.M. et al. (2019) Cytosolic Trapping of a Mitochondrial Heat Shock Protein Is an Early Pathological Event in Synucleinopathies. Cell Rep 28 (1), 65-77 e6.

34. Di Maio, R. et al. (2016) alpha-Synuclein binds to TOM20 and inhibits mitochondrial protein import in Parkinson's disease. Sci Transl Med 8 (342), 342ra78.

35. Cenini, G. et al. (2016) Amyloid beta-peptides interfere with mitochondrial preprotein import competence by a coaggregation process. Mol Biol Cell 27 (21), 3257-3272.

36. Mossmann, D. et al. (2014) Amyloid-beta peptide induces mitochondrial dysfunction by inhibition of preprotein maturation. Cell Metab 20 (4), 662-9.

37. Devi, L. et al. (2006) Accumulation of amyloid precursor protein in the mitochondrial import channels of human Alzheimer's disease brain is associated with mitochondrial dysfunction. J Neurosci 26 (35), 905768.

38. Ruan, L. et al. (2017) Cytosolic proteostasis through importing of misfolded proteins into mitochondria. Nature 543 (7645), 443-446.

39. Li, Y. et al. (2019) A mitochondrial FUNDC1/HSC70 interaction organizes the proteostatic stress response at the risk of cell morbidity. EMBO J 38 (3).

40. Richter-Dennerlein, R. et al. (2016) Mitochondrial Protein Synthesis Adapts to Influx of Nuclear-Encoded Protein. Cell 167 (2), 471-483 e10.

41. Levine, C.G. et al. (2005) The efficiency of protein compartmentalization into the secretory pathway. Mol Biol Cell 16 (1), 279-91.

42. Hansen, K.G. et al. (2018) An ER surface retrieval pathway safeguards the import of mitochondrial membrane proteins in yeast. Science 361 (6407), 1118-1122.

43. Matsumoto, S. et al. (2019) Msp1 Clears Mistargeted Proteins by Facilitating Their Transfer from Mitochondria to the ER. Mol Cell.

44. Itakura, E. et al. (2016) Ubiquilins Chaperone and Triage Mitochondrial Membrane Proteins for Degradation. Mol Cell 63 (1), 21-33.

45. Kundra, R. et al. (2017) Protein homeostasis of a metastable subproteome associated with Alzheimer's disease. Proc Natl Acad Sci U S A 114 (28), E5703-E5711.

46. Gruber, A. et al. (2018) Molecular and structural architecture of polyQ aggregates in yeast. Proc Natl Acad Sci U S A 115 (15), E3446-E3453.

47. Gidalevitz, T. et al. (2006) Progressive disruption of cellular protein folding in models of polyglutamine diseases. Science 311 (5766), 1471-4.

48. Walther, D.M. et al. (2015) Widespread Proteome Remodeling and Aggregation in Aging C. elegans. Cell 161 (4), 919-32.

49. Nargund, A.M. et al. (2015) Mitochondrial and nuclear accumulation of the transcription factor ATFS-1 promotes OXPHOS recovery during the UPR $(\mathrm{mt})$. Mol Cell 58 (1), 123-33.

50. Wrobel, L. et al. (2015) Mistargeted mitochondrial proteins activate a proteostatic response in the cytosol. Nature 524 (7566), 485-8.

51. Topf, U. et al. (2018) Quantitative proteomics identifies redox switches for global translation modulation by mitochondrially produced reactive oxygen species. Nat Commun 9 (1), 324. 
52. Liu, W. et al. (2018) Mitochondrial protein import regulates cytosolic protein homeostasis and neuronal integrity. Autophagy 14 (8), 1293-1309.

53. Poveda-Huertes, D. et al. (2019) An Early mtUPR: Redistribution of the Nuclear Transcription Factor Rox1 to Mitochondria Protects against Intramitochondrial Proteotoxic Aggregates. Mol Cell.

54. Wang, X. and Chen, X.J. (2015) A cytosolic network suppressing mitochondria-mediated proteostatic stress and cell death. Nature 524 (7566), 481-4.

55. Rolland, S.G. et al. (2019) Compromised Mitochondrial Protein Import Acts as a Signal for UPR(mt). Cell Rep 28 (7), 1659-1669 e5.

56. Labbadia, J. et al. (2017) Mitochondrial Stress Restores the Heat Shock Response and Prevents Proteostasis Collapse during Aging. Cell Rep 21 (6), 1481-1494.

57. Heo, J.M. et al. (2010) A stress-responsive system for mitochondrial protein degradation. Mol Cell 40 (3), 465-80.

58. Wu, Z. et al. (2019) MISTERMINATE Mechanistically Links Mitochondrial Dysfunction with Proteostasis Failure. Mol Cell 75 (4), 835-848 e8.

59. Izawa, T. et al. (2017) Cytosolic Protein Vms1 Links Ribosome Quality Control to Mitochondrial and Cellular Homeostasis. Cell 171 (4), 890-903 e18.

60. Su, T. et al. (2019) Structure and function of Vms1 and Arb1 in RQC and mitochondrial proteome homeostasis. Nature 570 (7762), 538-542.

61. Zurita Rendon, O. et al. (2018) Vms1p is a release factor for the ribosome-associated quality control complex. Nat Commun 9 (1), 2197.

62. Haynes, C.M. et al. (2007) ClpP mediates activation of a mitochondrial unfolded protein response in C. elegans. Dev Cell 13 (4), 467-80.

63. Nargund, A.M. et al. (2012) Mitochondrial import efficiency of ATFS-1 regulates mitochondrial UPR activation. Science 337 (6094), 587-90.

64. Quiros, P.M. et al. (2017) Multi-omics analysis identifies ATF4 as a key regulator of the mitochondrial stress response in mammals. J Cell Biol 216 (7), 2027-2045.

65. Fiorese, C.J. et al. (2016) The Transcription Factor ATF5 Mediates a Mammalian Mitochondrial UPR. Curr Biol 26 (15), 2037-2043.

66. Kim, H.E. et al. (2016) Lipid Biosynthesis Coordinates a Mitochondrial-to-Cytosolic Stress Response. Cell 166 (6), 1539-1552 e16.

67. Hahn, J.S. and Thiele, D.J. (2004) Activation of the Saccharomyces cerevisiae heat shock transcription factor under glucose starvation conditions by Snf1 protein kinase. J Biol Chem 279 (7), 5169-76.

68. Murphy, J.P. et al. (2015) Comprehensive Temporal Protein Dynamics during the Diauxic Shift in Saccharomyces cerevisiae. Mol Cell Proteomics 14 (9), 2454-65.

69. Matilainen, O. et al. (2017) The chromatin remodeling factor ISW-1 integrates organismal responses against nuclear and mitochondrial stress. Nat Commun 8 (1), 1818.

70. Krakowiak, J. et al. (2018) Hsf1 and Hsp70 constitute a two-component feedback loop that regulates the yeast heat shock response. Elife 7.

71. Masser, A.E. et al. (2019) Cytoplasmic protein misfolding titrates Hsp70 to activate nuclear Hsf1. Elife 8.

72. Hahn, J.S. et al. (2006) A stress regulatory network for co-ordinated activation of proteasome expression mediated by yeast heat shock transcription factor. Mol Microbiol 60 (1), 240-51.

73. Schmidt, R.M. et al. (2019) The proteasome biogenesis regulator Rpn4 cooperates with the unfolded protein response to promote ER stress resistance. Elife 8.

74. Wang, X. et al. (2008) Reduced cytosolic protein synthesis suppresses mitochondrial degeneration. Nat Cell Biol 10 (9), 1090-7.

75. Munch, C. and Harper, J.W. (2016) Mitochondrial unfolded protein response controls matrix pre-RNA processing and translation. Nature 534 (7609), 710-3.

76. Samluk, L. et al. (2019) Cytosolic translational responses differ under conditions of severe short-term and long-term mitochondrial stress. Mol Biol Cell 30 (15), 1864-1877.

77. Baker, B.M. et al. (2012) Protective coupling of mitochondrial function and protein synthesis via the elF2alpha kinase GCN-2. PLoS Genet 8 (6), e1002760.

78. Borch Jensen, M. et al. (2017) PGAM5 promotes lasting FoxO activation after developmental mitochondrial stress and extends lifespan in Drosophila. Elife 6. 
79. Labbadia, J. and Morimoto, R.I. (2015) Repression of the Heat Shock Response Is a Programmed Event at the Onset of Reproduction. Mol Cell 59 (4), 639-50. 80. Merkwirth, C. et al. (2016) Two Conserved Histone Demethylases Regulate Mitochondrial StressInduced Longevity. Cell 165 (5), 1209-1223. 81. Tian, Y. et al. (2016) Mitochondrial Stress Induces Chromatin Reorganization to Promote Longevity and UPR(mt). Cell 165 (5), 1197-1208.

631 82. Lo, S.C. and Hannink, M. (2008) PGAM5 tethers a ternary complex containing Keap1 and Nrf2 to 632 mitochondria. Exp Cell Res 314 (8), 1789-803.

633 83. Tsakiri, E.N. et al. (2013) Proteasome dysfunction in Drosophila signals to an Nrf2-dependent regulatory circuit aiming to restore proteostasis and prevent premature aging. Aging Cell 12 (5), 802-13.

635 84. Lehrbach, N.J. and Ruvkun, G. (2019) Endoplasmic reticulum-associated SKN-1A/Nrf1 mediates a cytoplasmic unfolded protein response and promotes longevity. Elife 8.

637

638 85. Jin, S.M. et al. (2010) Mitochondrial membrane potential regulates PINK1 import and proteolytic destabilization by PARL. J Cell Biol 191 (5), 933-42.

639 86. Burman, J.L. et al. (2017) Mitochondrial fission facilitates the selective mitophagy of protein aggregates. J Cell Biol 216 (10), 3231-3247.

641 87. Jin, S.M. and Youle, R.J. (2013) The accumulation of misfolded proteins in the mitochondrial matrix is sensed by PINK1 to induce PARK2/Parkin-mediated mitophagy of polarized mitochondria. Autophagy 9 (11), 1750-7. 88. Mutka, S.C. and Walter, P. (2001) Multifaceted physiological response allows yeast to adapt to the loss of the signal recognition particle-dependent protein-targeting pathway. Mol Biol Cell 12 (3), 577-88. 89. Guo, X. et al. (2019) Mitochondrial dysfunction is signaled to the integrated stress response by OMA1, DELE1 and HRI. bioRxiv, 715896. 